\title{
Basic movements for postoperative exercise in patients with left ventricular assist devices
}

\author{
Massimiliano Polastri ${ }^{1}$, Giulia Zagnoni ${ }^{1}$, Antonio Loforte ${ }^{2}$ \\ ${ }^{1}$ Medical Department of Continuity of Care and Disability, Physical Medicine and Rehabilitation, University Hospital \\ St. Orsola-Malpighi, Bologna; ${ }^{2}$ Department of Cardiac-Thoracic and Vascular Diseases, Cardiac Surgery and \\ Transplantation, University Hospital St. Orsola-Malpighi, Bologna, Italy
}

\begin{abstract}
Patients with advanced heart failure refractory to medical therapy can be treated with left ventricular assist devices, implanted to augment or replace left ventricular function. The most common postoperative complications are infection; bleeding; thromboembolic events; device malfunction; depression, and neurological dysfunction (stroke, transient ischemic attacks, encephalopathy). Their onset contributes to the interruption and delay in commencing a physiotherapy programme. During the initial postoperative phase, patients with left ventricular assist devices are not generally able to tolerate an intensive rehabilitative programme, but they may be able to do so in a more advanced stage of recovery. Physiotherapy in the early period prepares the patient for a more complex and articulated rehabilitation, usually scheduled after hospital discharge. Supervised exercise should be implemented once the patient has been thoroughly instructed on how the device works and after a checklist to ensure that the patient understands the workings of the device, has been signed off. Although several studies have been published discussing postoperative rehabilitation in LVAD patients, to date it is not available yet an illustrated guide describing basic movements. The current study aims at illustrating a set of basic
\end{abstract}

Correspondence: Massimiliano Polastri, Medical Department of
Continuity of Care and Disability, Physical Medicine and
Rehabilitation, University Hospital St. Orsola-Malpighi,
Via G. Massarenti 9, Bologna 40138, Italy.
Tel. +39.051.2144980 - Fax +39.051.0822185.
E-mail: gbptap1@gmail.com
Conflict of interest: The authors have no conflict of interest to declare.

Key words: Exercise therapy; left ventricular assist devices; postoperative care; rehabilitation.

Received for publication: 28 August 2018.

Accepted for publication: 28 Janaury 2019.

(C) Copyright M. Polastri et al., 2019

Licensee PAGEPress, Italy

Monaldi Archives for Chest Disease 2019; 89:995

doi: 10.4081/monaldi.2019.995

This article is distributed under the terms of the Creative Commons Attribution Noncommercial License (by-nc 4.0) which permits any noncommercial use, distribution, and reproduction in any medium, provided the original author(s) and source are credited. movements preparatory to a more complex and articulated exercise programme. A range of exercises involving all body parts is described as lying, sitting and standing postures.

\section{Introduction}

After left ventricular assist device (LVAD) implantation, physiotherapy administered to patients does not substantially differ from that recommended for cardiac surgery patients, as the primary goal is the treatment/prevention of postoperative pulmonary complications (especially during the early postoperative phase), and the enhancement of motor activities [1,2]. However, a significant difference between cardiac surgery patients (i.e., coronary artery bypass grafting, valve replacement) and patients with an LVAD can be recognised in the complexity of the general preoperative conditions, as the latter patients are more prone to physical deconditioning because of forced bed rest and physical inactivity. Another substantial difference is the management of the LVAD itself, by either the patient or his/her caregivers. Then, principal differences between the usual cardiac surgery patients and LVAD recipients are the less demanding clinical complexity of cardiac surgical procedures, and the need in patients with an LVAD to make an additional effort to understand the functioning of the device and to manage it.

The importance of early mobilisation and assessment of the overall physical condition of both LVAD recipients and other classes of patients are solid concepts for those physiotherapists involved in the recovery pathway of cardiac-thoracic surgical patients [1,3-9]. Early mobilisation, which is particularly effective in patients whose postoperative courses have been complicated [1], optimises recovery before heart transplantation [5]. In other reports in the literature, exercise was initiated as early as possible after LVAD implantation, initially starting in the intensive care unit (ICU) and lasting just a few minutes, with gradual progression up to 30-40 min, 3-5 days a week [10]. Moreover, exercise was generally not linked to the development of adverse effects, and the few such cases were attributed to an acute decrease in pump flow $[5,10]$. The studies showed that patients with an LVAD were prone to physical deconditioning because of their preoperative conditions, but early motor activity programs were safe and feasible. Early activities consisted principally of in-bed positioning, postural transfers (from the bed to a chair to a standing position), ambulation, active exercises, walking, and stair-climbing, with exercise intensity increasing over time. Supervised exercise should be implemented once the patient has been fully instructed on how the device works after his/her understanding has been tested with a positive result. 
Although several studies have been published discussing postoperative rehabilitation in LVAD patients, to date it is not available yet an illustrated guide. With the current article we aimed at illustrating a set of basic movements preparatory to a more complex and articulated exercise programme; a range of exercises involving all body parts was described as lying, sitting and standing postures.

The supervised exercises illustrated here can be executed safely by LVAD patients as early as possible during the initial in-hospital postoperative phase following device implantation.

\section{Supervised exercise}

Postoperative motor recovery is usually started in the ICU and continued during the hospital stay focusing on mobility, progressive mobilisation, strengthening exercises, cycling, and ambulation [10], as previously discussed. Active mobilisation and walking have been included as a component of the inpatient rehabilitation in a cohort of LVAD recipients [11]. Exercising in ICU is dependent on clinical conditions, the medications regimen, inotropic agent infusion status, Swan-Ganz catheterisation status, and hemodynamic stability.

If patients with an LVAD have been bedridden for a long time before implantation or are debilitated, or if a complicated postoperative recovery course has created a need for prolonged ICU stay, it is likely that reduced motor capacity will also be observed during the performance of basic movements such as effective trunk control or postural transfers. On the other hand, while safety is essential from the physiotherapist's perspective, exercise progression should be based around a collaborative approach between the physiotherapist encouraging and educating the patient on graduated exercise and the patient's willingness to comply.

\section{Movements characteristics}

Some exercises aiming to involve all the body segments have been selected here. They are grouped according to the starting position as follows: a supine position on the bed, a sitting position at the edge of the bed, and an upright position. As previously discussed by de Jonge et al. [7], the aim of physical activities during the first postoperative weeks -after LVADs implantation- is to get the patient accustomed to exercise. It has been demonstrated that in patients with chronic heart failure, exercise performance is also related to skeletal muscle abnormalities including atrophy [7,12].

Considering these findings, we chose a set of exercises whose characteristics allow at early patient mobilisation. Prescribing physical training in patients with an LVAD should reflect the patient's progression along the recovery pathway; we were inspired by the challenges faced in the daily clinical practice while providing physiotherapy in such class of patients.

We selected those movements that: 1) guarantee the maximum degree of safety; 2) can be executed involving all body segments; 3) can be performed at the bedside; 4) can be quickly learned by patients; 5) facilitates patients to undertaking some responsibility for self-management when they have an understanding of the benefits of exercise; 6) can allow for wide choice of positions; 7) can be adapted to different stages of hospitalization; 8) can be standardised and reproduced; 9) provides for variable duration to best meet the characteristics of each patient; and 10) contributes to improving equilibrium as patients with an LVAD are required to wear a bag to carry the controller and batteries.
Movements illustrated here are thus mainly directed to enhance lower body mobility and strengthening, because muscle dysfunctions in patients with chronic heart failure [13] are likely to affect primarily the muscle groups that work against gravity, such as quadriceps and gluteus. In light of these assumptions, the following exercises can contribute to achieving safely early mobility in patients with an LVAD.

\section{Frequency, intensity, and duration}

Frequency (number of days per week) of exercise can vary depending on patient's condition, however, in a cohort study, patients with an LVAD were able to tolerate three hours daily of physiotherapy for six days per week [14]. In addition, in a recent Cochrane review by Anderson et al. [15] that investigated exercise frequency in patients who underwent coronary artery bypass grafting, it has been confirmed that exercise prescription can vary considerably ( 1 to 7 sessions per week).

The possibility to perform exercise could also be related to the availability of a physiotherapy service [16]; generally, in acute/sub-acute settings physiotherapy is available 6 to 7 days per week. However, in a previously published systematic review, exercise frequency in patients with an LVAD ranged from 3 to 5 days per week [10]. In another recent study by Racca et al. [17] exercise sessions reached a maximum of $50 \mathrm{~min}$ and were performed twice a day for six days a week.

Determination of exercise intensity (difficulty) can be achieved using the Borg scale, which is commonly administered to assess exercise intensity in cardiac patients $[10,18]$, limiting the effort to light/somewhat hard, as confirmed in the literature $[7,10,16,19]$. As the exercises illustrated here are designed to be executed during the initial postoperative phase, their intensity can also be tailored referring to vital parameters as in this timeframe (sub-acute setting) patients with an LVAD are typically monitored continuously. It has been reported that $50-70 \%$ of peak heart rate and mean arterial pressure $70-95 \mathrm{mmHg}$ can be used as exercise parameters in addition to the use of the Borg scale [10,17].

A large body of evidence supports that duration (time) of exercises in patients with an LVAD throughout the early postoperative recovery range between $20-50$ minutes per session $[10,17,20]$. If all exercises illustrated here are performed in one single session, its duration would be $30-40 \mathrm{~min}$ (performing approximately five repetitions for each position). Exercise sessions were designed to be repeated once to twice a day according to the demonstrated possibility for LVAD patients to tolerate relative intense training [14]. Ideally, workouts can be divided into two daily sessions -morning and afternoon- whose duration can be scheduled to reach progressively more intensity [14] allowing patients to take some hours of rest. Dividing sessions into two or more moments can also facilitate other interventions not directly related to physiotherapy (nursing and medical activities). The number of repetitions, frequency, and duration should be of course adapted to increase progressively throughout the hospital stay. Progressive independent ambulation and cycling can be initiated in parallel within the first 15 postoperative days as already described in previously published studies $[5,10,16]$. Synthesising all the above considerations, the supervised movements illustrated here can be performed 5 to 6 days per week and can be divided into two daily sessions (Borg scale light/somewhat hard) whose duration should be about $1 \mathrm{~h}$ each, to get the patient accustomed to exercise within the first postoperative weeks. 


\section{Involvement of the upper limbs}

Prevention of postoperative sternal complications following cardiac surgery, when performed via median sternotomy, is routinely achieved by placing restrictions on the use of the upper limbs such as reducing the range of motion and load applied; physiotherapist habitually introduce these restrictions in the acute setting particularly on unilateral elevation, lifting, and weight load [21,22].

Nevertheless, in a recent randomised controlled trial protocol, it has been hypothesised that upper limbs movements are possible using both arms for exercises and activities [23]; at the same time, there is a lack of consensus on postoperative upper limbs exercise restrictions [21]. The safety score of the movements of the upper extremities illustrated here is high in accordance with the findings by Adams et al. [24]: 3 on a scale ranging between 3 to 1 (where 3 represents the absence of risk and 1 the highest risk) assuming, therefore, no risk for sternal healing.

\section{Notes to the illustrations}

For illustrative purposes, a model rather than a patient has been used to demonstrate the position and movements, and thus the images contained here do not include the driveline connecting the device to the patient: the illustrated bag serves merely as an example. During the execution of the exercises, the device must be positioned safely and stable with no tension in the driveline.

\section{Exercises in a supine position}

Exercises performed in a supine position aim to strengthen muscular groups most prone to atrophy because of non-use, including the gluteus and quadriceps muscles. The main advantage of exercises performed when supine is that they can be commenced in the early phase of postoperative recovery, thereby minimising clinical risks (falls, exercise-related injuries) and reducing mechanical stress. Supine exercises can be used either as warm-ups or as components of more complex motor programmes. Typically, these movements can be executed as soon as the patient is cooperative, but the presence of lines, drainages, and/or catheters must be considered at all time to avoid exercise-induced damages. The safety of the patient is guaranteed by the position itself and by the bed's retaining bars when needed (Figure 1). Progression toward a different posture (sitting, upright) should be encouraged when the patient is comfortable with supine exercise training, and no adverse events have occurred.
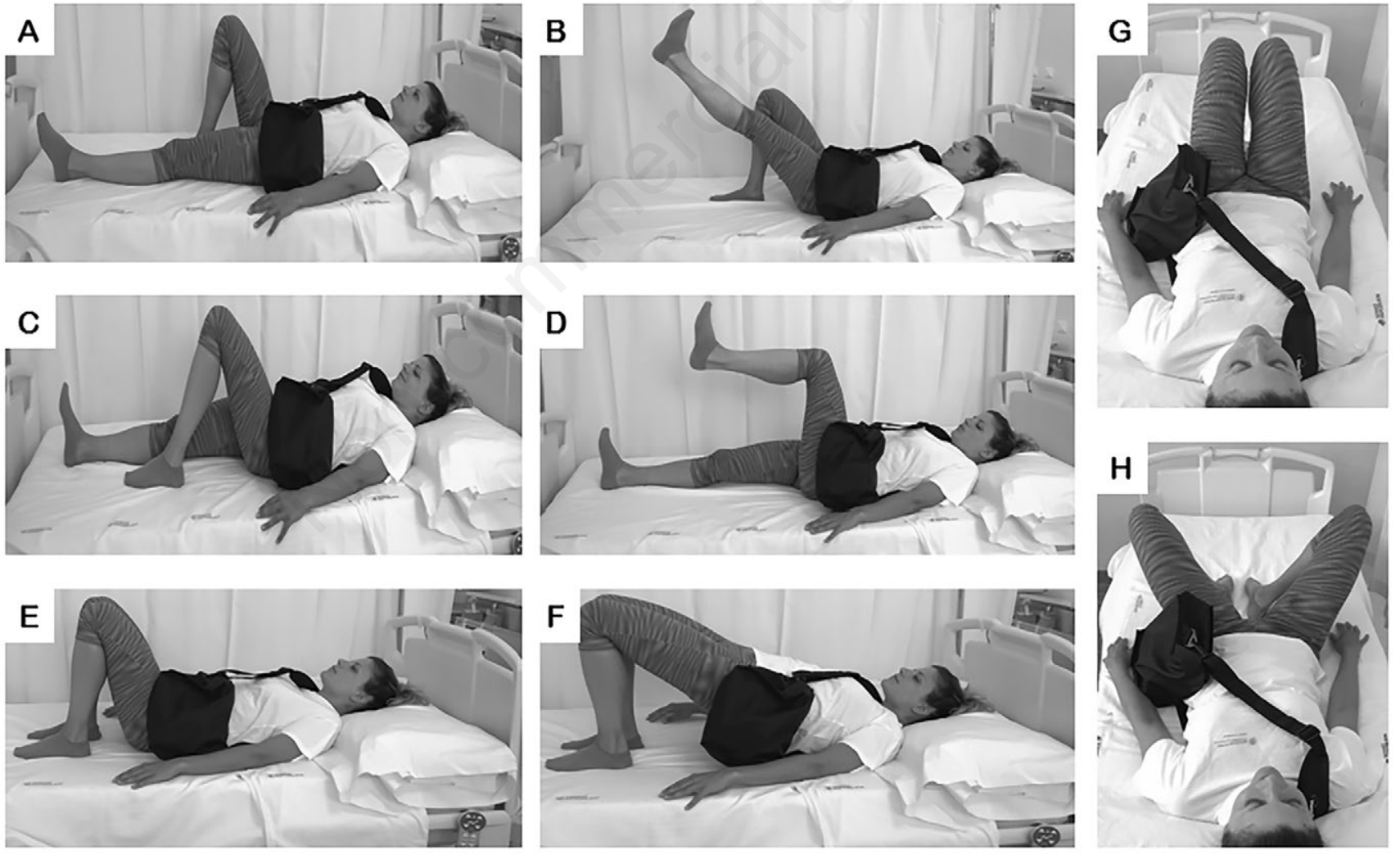

Figure 1. Exercises performed in a supine position. A) The patient lies supine in the centre of the bed and bends the right leg with the sole on the surface of the bed. B) While exhaling, the left leg is extended upward, and the position held for about 3 s; the same movement is then performed on the opposite side. C) The patient lies supine in the centre of the bed. Keeping the right leg straight, the left leg is bent upward with the sole held on the surface of the bed. D) While exhaling, the left leg is bent up toward the chest, and the hip and knee are held at $90^{\circ}$ for about $3 \mathrm{~s}$; the same movement is then performed on the opposite side. E) The patient lies supine in the centre of the bed; both legs are bent with the soles kept on the surface of the bed. F) While exhaling, the buttocks are raised off the bed as far as possible, and the position is held for 3-5 s. G) The patient lies supine in the centre of the bed; both legs are bent with the soles kept on the surface of the bed. $H$ ) While exhaling, knees are maximally separated. 


\section{Exercises in a sitting position at the edge of the bed or on a chair}

During sitting exercises, an antigravity posture is actively preserved. This aids in the development of the strength required to maintain the physical position against the force of gravity. Once the patient can transition safely from a supine to a sitting position, incremental increases in the exercises performed in the sitting position should be encouraged with the aim of achieving better trunk control (Figures 2 and 3). To avoid driveline tension and/or entrapment, these exercises should be performed when the patient is entirely cooperative, and a certain degree of autonomy has been reached. Progression toward upright supervised training should be allowed only when the patient is confident in the ability to perform the sitting exercises, and no adverse events have occurred. Arms movements are performed maintaining the arms close to the body $[21,22]$ (Figure 2).

\section{Transitioning from a sitting to an upright position and walking in place}

The transition from sitting to a standing position allows the strengthening of more than one muscle group with a single move- ment (Figure 4). The aims are to improve functional capacity and personal autonomy. This exercise can be introduced when the patient gains strength but is not yet able to leave the chair/bed easily. The patient should be supervised during the performance of the exercise, to avoid driveline impingements. The exercise can be accompanied by walking in place to enhance body flexibility and equilibrium (Figure 4).

\section{Exercises in an upright position and walking}

When postural transitions from the upright position and in-bed movements become autonomous, the patient is ready to walk (sometimes a frame may be temporarily required by patients with complications). Walking, standing on tip-toes, bending the knees, cycling, stair-climbing, and other exercises must all be supervised.

Walking, bicycle and treadmill are among the most advanced types of training for patients with an LVAD particularly during the outpatient rehabilitation phase: duration of exercise has been found to increase gradually from 20 to 40 minutes per session $[11,16,17]$. Exercises in an upright position (Figure 5) are an essential component of muscular training, specifically, the enhancement of muscular strength and the optimisation of equilibrium. The patient should be encouraged to perform these exercises when he/she can walk, and the first independent activities are possible. Walking, cycling
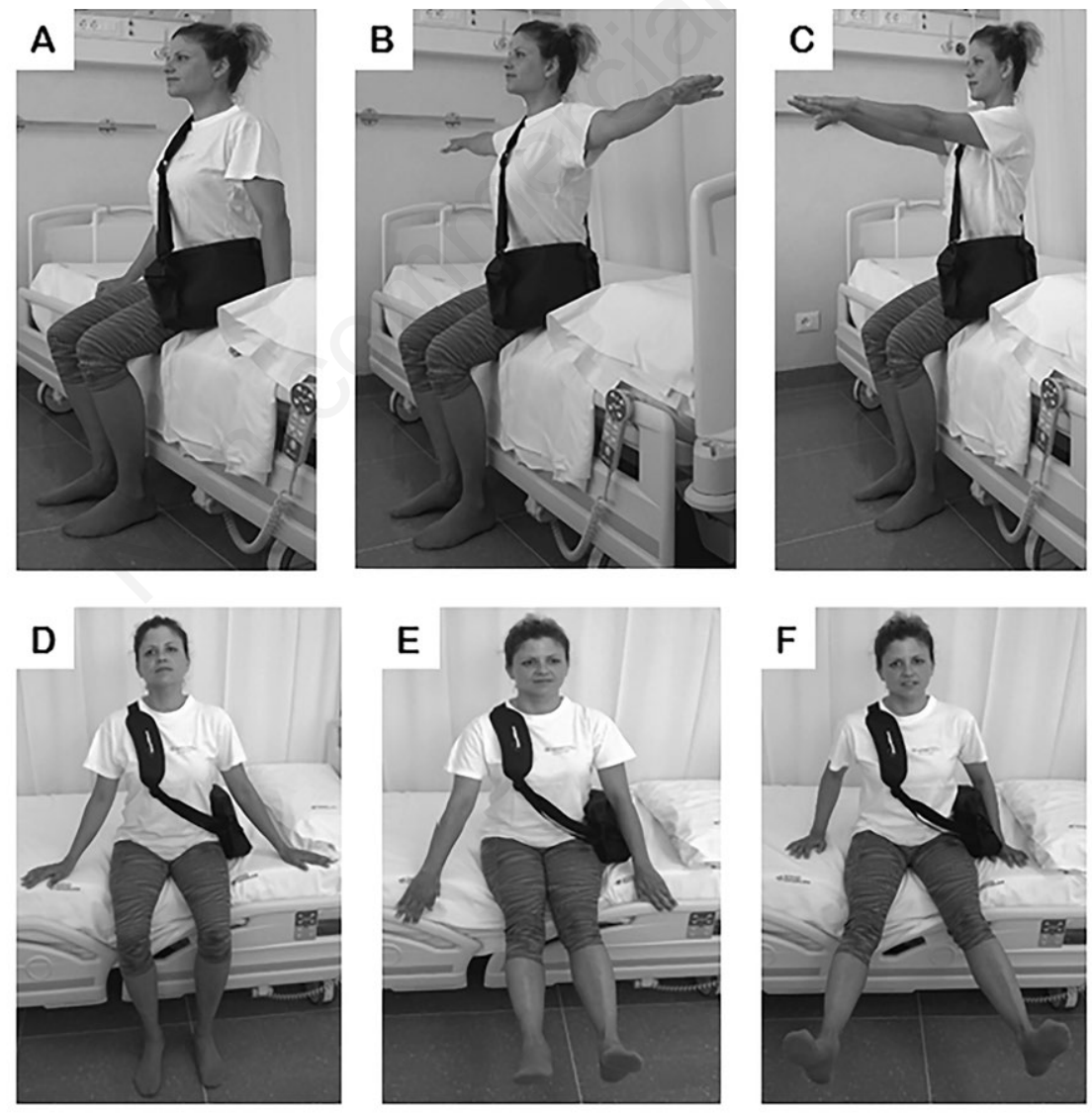

Figure 2. Exercises performed in a sitting position. A) The patient sits on the edge of the bed or a chair with the soles on the floor for about $5 \mathrm{~s}$ and then relaxes. B) The arms are raised, and the position held for about $5 \mathrm{~s}$. C) The arms are extended to the front, and the pose held for about $5 \mathrm{~s}$. D-F) The patient sits on the edge of the bed or a chair with the soles on the floor; both knees are fully extended; legs are separated, and the position maintained with the aid of hand support. 
and stair-climbing (Figure 5) should be integrated with other exercises and should be commenced as soon as the patient can maintain upright postural control.

\section{Exercise precautions}

During rehabilitation activities and physiotherapy, it is possible that sudden hemodynamic changes occur in patients with an LVAD, thus is essential that patients are monitored closely to detect adverse physiological events. Exercise should be interrupted and even avoided if significant alterations in the cardiovascular system occur (Figure 6), if the patient develops chest pain or palpitations, or if the oxygen saturation is $<90 \%$, as discussed in a previous systematic review [10]. In general, before patients are allowed to engage in exercise, it must be confirmed that there are no contraindications posed by their overall clinical condition
$[25,26]$. In order to commence the supervised exercise in the initial phase during the ICU stay, cooperating patients must be weaned from mechanical ventilation and be hemodynamically stable. Before starting the supervised exercises, the following functional indicators should be considered: the patient can maintain a sitting position free of symptoms for at least $1 \mathrm{~h} 30$ ', the patient is able to maintain a standing position, and he/she can walk a few meters [27]. Although the movements we have illustrated here can be initiated already with the patient in the ICU, the use of the indicators listed above can certainly optimise the execution of the exercises during the phase of the sub-intensive care setting. Monitoring of perceived effort, dyspnea, and the vital parameters is essential during physiotherapeutic sessions. Exercise intolerance in patients with an LVAD can be elicited by contributing factors such as fixed continuous flow-LVAD pump speed, sarcopenia, abnormal muscular response to vasodilation, aortic valve abnormalities, anaemia, ventilation/perfusion mismatch, reduced right ventricular function [28]. In LVAD patients is also crucial to maintain an optimal circu-
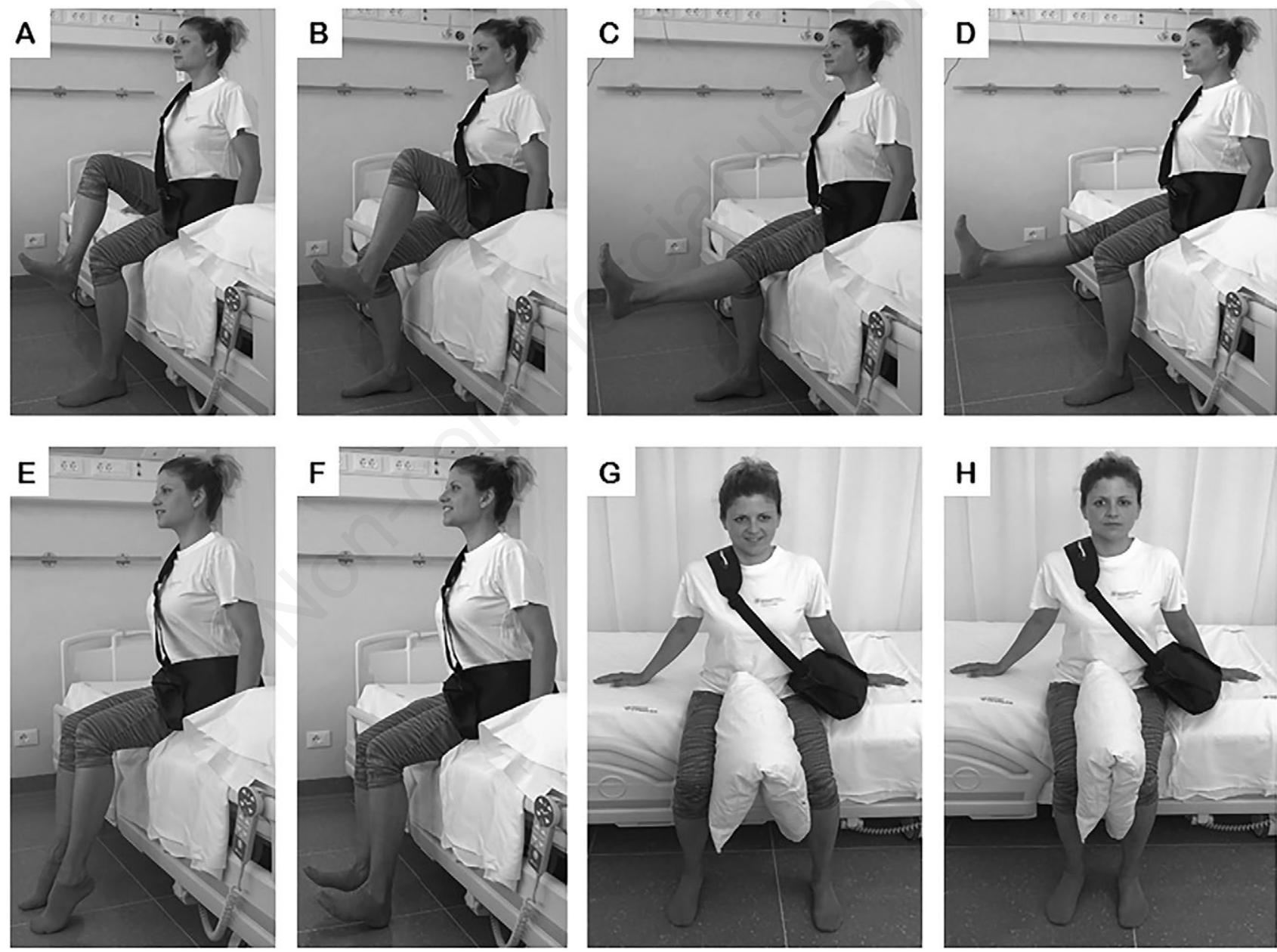

Figure 3. Exercises performed in a sitting position. A) The patient sits on the edge of the bed or a chair with the soles on the floor and the palms of the hands on the bed, and then bends the right leg toward the chest to the maximal extent and holds the position for about $3 \mathrm{~s}$. B) The same movement is repeated on the opposite side. C) The patient sits on the edge of the bed or on a chair with the soles on the floor and the palms on the bed, and then entirely extends the right knee with the ankle maximally flexed; the pose is held for about 3 s. D) The same movement is repeated on the opposite side. E) The patient sits on the edge of the bed or a chair with the soles on the floor and then lifts the heels off the floor and holds the position for about $3 \mathrm{~s}$. F) After raising the heels, the forefeet are bent upwards and the position held for about $3 \mathrm{~s}$. G) The patient sits on the edge of the bed or a chair with the soles on the floor and squeezes a pillow between the knees. H) While exhaling, the pillow is squeezed between the knees. 
lating volume to avoid hypotension during physical activity, and suction effects on interventricular septum; during and after exercise patients should be stimulated to introduce small amounts of fluids to avoid hypovolemia [29]. If exercise-induced hemodynamic complications occur, the exercise must be interrupted, and the patient referred to the ward staff for physician evaluation. Exercise can be, afterwards, reinitiated as soon as the patient returns to stable clinical conditions and by following the criteria previously described. As we have formerly highlighted in the current article, the patient must be instructed on how to avoid driveline tension and how to carry the bag containing the controller and batteries, safely. Supervised-exercise at an early stage should be planned within the VAD multidisciplinary team to clarify the intended goals, and to identify potentially harmful details.

\section{Conclusions}

LVAD recipients are often convinced that the device implantation can negatively interact with their degree of mobility and that the device would affect their eligibility for transplantation [30]; considering these findings there are not substantial differences between those patients who are referred to LVAD as destination therapy (DT) and those who are implanted for a bridge to transplantation (BTT). In either case, improving physical function is indeed of primary importance; early mobilisation and supervised exercises have the same rationale in both DT and BTT patients. These assumptions seem to be also supported by a recent metaanalysis whose results showed no difference in 1-year mortality rates between patients implanted as BTT and those as DT vs heart transplantation [31]. Nevertheless, LVAD implantation is not free from complications such as psychological issues for patients and surrounding relatives, infections, neurologic dysfunctions, gastrointestinal bleeding, and thrombosis [32].

The implementation of an early mobilisation programme in patients with an LVAD can be undoubtedly enhanced by a multidisciplinary approach where all professionals are contributing to the pathway of care (cardiologist, physiotherapist, cardiac surgeon, nutritionist, psychologist, nurse) [27]. Following LVAD implantation supervised exercise should be initiated as soon as possible, after the patient has been thoroughly instructed on how the device works, and after a checklist to ensure that the patient understands the workings of the device, has been signed off. In this regard, the
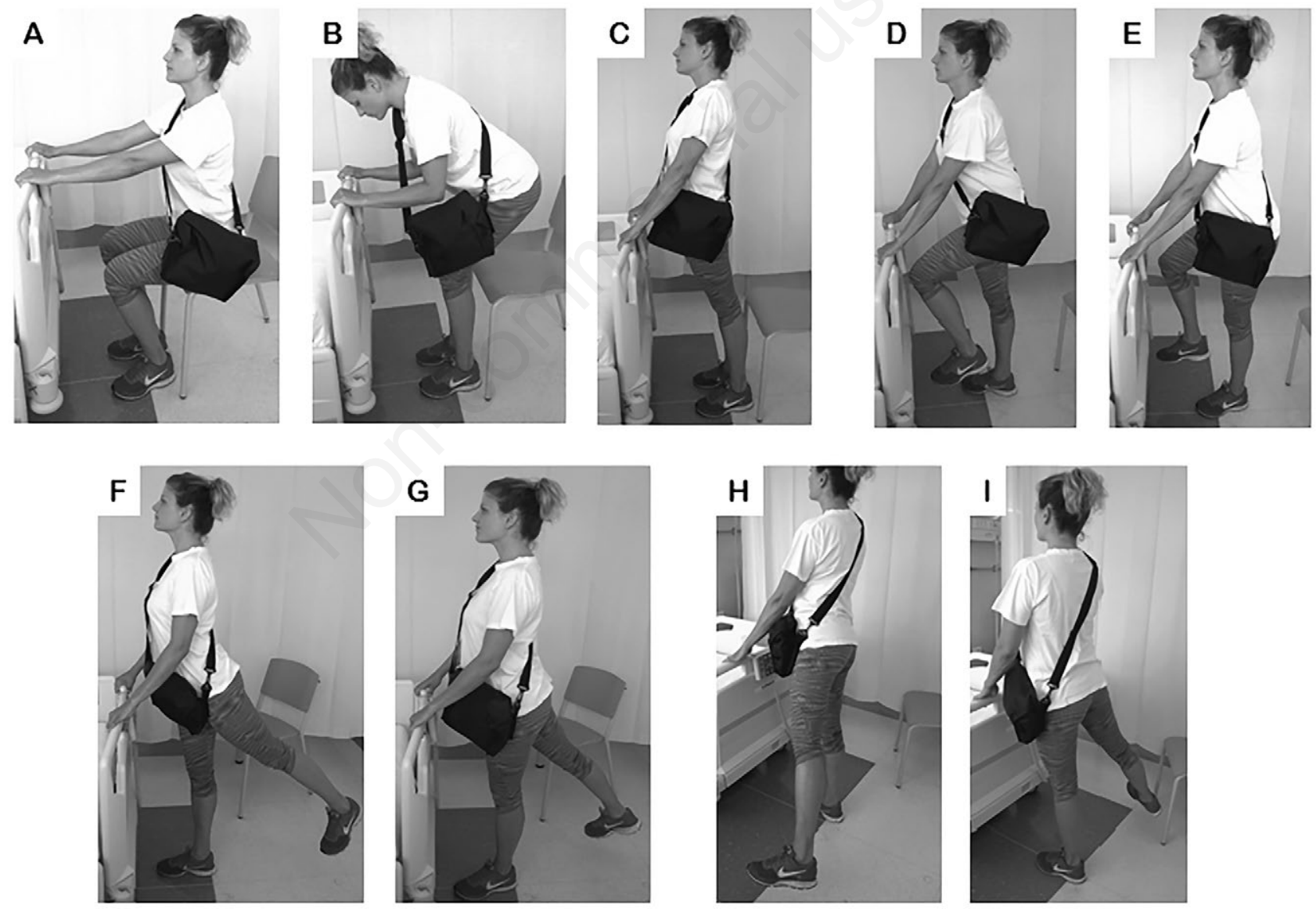

Figure 4. Exercises performed in an upright position. A) The patient sits on a chair with the soles on the floor and extends the hands to the bed. B) With the head forward, the patient begins to rise. C) The knees are fully extended, and the head straightened until the upright position is attained. D) The patient stands in front of the bed, extends the hands to the bed, and starts by lifting the left foot. E) The patient marches in place for as long as possible. F) The patient stands in front of the bed, rests the hands on the bed, and extends the left leg backwards. G) This exercise is repeated for the opposite side. H) The patient stands in front of the bed, brings the hands to the bed, and extends the left leg laterally. I) This exercise is repeated for the opposite side. 
patient must be able to substitute the batteries and to understand the controller alarms, and he/she can appropriately interact with the members of the multidisciplinary team [27]. A skilled professional must supervise the movements illustrated here, and a certain degree of progression must be expected during the postoperative recovery. Aiming at illustrating those movements suitable for the postoperative exercise in LVAD patients, we must not forget that safety is of primary importance and one must be aware that exercise must be supervised at all time: to date, there is no evidence supporting self-administered exercise in patients with an LVAD.

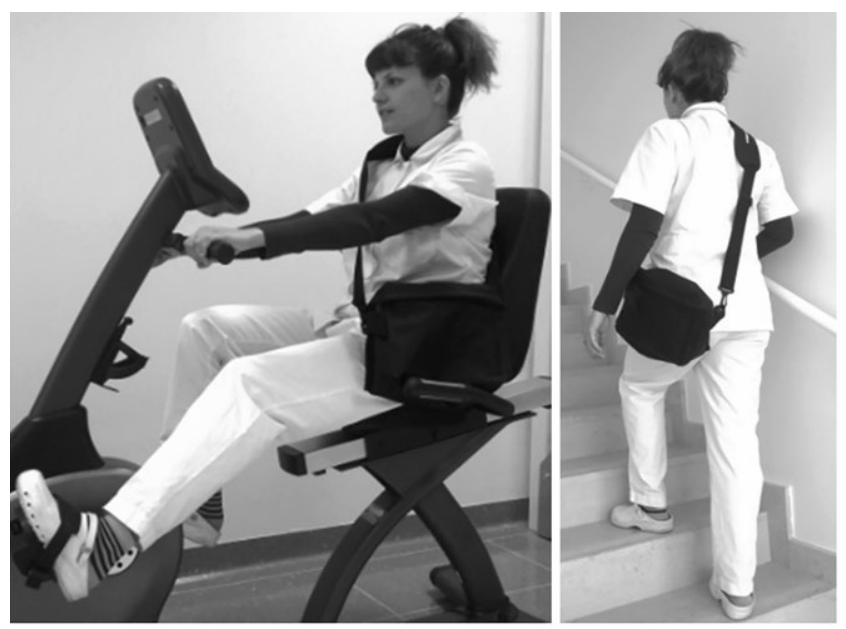

Figure 5. Cycling and climbing stairs wearing the LVAD pack. The device must be positioned safely and stable with no tension in the driveline while cycling. Posterior back support can facilitate exercise execution allowing a more comfortable and secure device positioning.
Although the many technical advances and improved knowledge of the physiology imposed by LVADs have paved the way for an active postoperative pathway of care, early physiotherapy for patients with an LVAD remain of primary importance.

\section{Acknowledgements}

The Authors wish to thank Dr. Leonida Compostella for his contribution.

\section{References}

1. Perme CS, Southard RE, Joyce DL, et al. Early mobilization of LVAD recipients who require prolonged mechanical ventilation. Tex Heart Inst J 2006;33:130-3.

2. Yost G, Coyle L, Milkevitch K, et al. Efficacy of inpatient rehabilitation after left ventricular assist device implantation. PM R 2017;9:40-5.

3. Schweickert WD, Kress JP. Implementing early mobilization interventions in mechanically ventilated patients in the ICU. Chest 2011;140:1612-7.

4. Granegger M, Schlöglhofer T, Ober H, et al. Daily life activity in patients with left ventricular assist devices. Int J Artif Organs 2016;39:22-7.

5. Morrone TM, Buck LA, Catanese KA, et al. Early progressive mobilization of patients with left ventricular assist devices is safe and optimizes recovery before heart transplantation. J Heart Lung Transplant 1996;15:423-9.

6. Humphrey R. Exercise physiology in patients with left ventricular assist devices. J Cardiopulm Rehabil 1997;17:73-5.

7. de Jonge N, Kirkels H, Lahpor JR, et al. Exercise performance in patients with end-stage heart failure after implantation of a
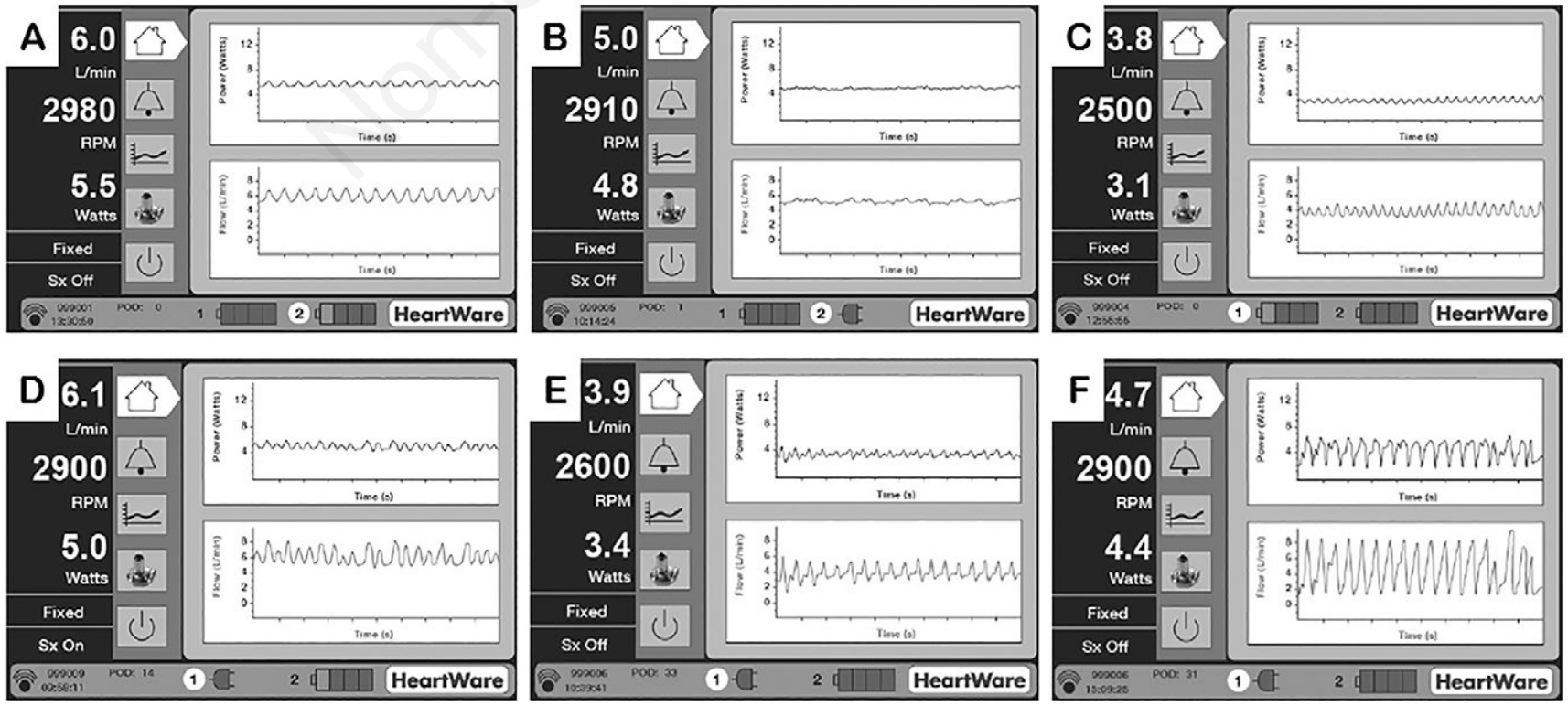

Figure 6. Device readouts (examples). A) Normal operation curve. B) Hypovolemia; low systemic mean arterial pressure; excessive pump speed. C) Ventricular tachycardia. D) Arrhythmias (irregular heart rate). E,F) Suction. Reproduced with permission of Medtronic, Inc. 
left ventricular assist device and after heart transplantation: an outlook for permanent assisting? J Am Coll Cardiol 2001;37: 1794-9.

8. Stiller K. Physiotherapy in intensive care: an updated systematic review. Chest 2013;144:825-47.

9. Wahab R, Yip NH, Chandra S, et al. The implementation of an early rehabilitation program is associated with reduced length of stay: a multi-ICU study. J Intensive Care Soc 2016;17:2-11.

10. Scheiderer R, Belden C, Schwab D, et al. Exercise guidelines for inpatients following ventricular assist device placement: a systematic review of the literature. Cardiopulm Phys Ther J 2013;24:35-42.

11. Lamotte MX, Chimenti S, Deboeck G, et al. Left ventricular assist device: exercise capacity evolution and rehabilitation added value. Acta Cardiol 2018;73:248-55.

12. Mancini DM, Walter G, Reichek N, et al. Contribution of skeletal muscle atrophy to exercise intolerance and altered muscle metabolism in heart failure. Circulation 1992;85:1364-73.

13. George I, Xydas S, Mancini DM, et al. Effect of clenbuterol on cardiac and skeletal muscle function during left ventricular assist device support. J Heart Lung Transplant 2006;25:1084-90.

14. Nguyen E, Stein J. Functional outcomes of adults with left ventricular assist devices receiving inpatient rehabilitation. PM R 2013;5:99-103.

15. Anderson L, Thompson DR, Oldridge N, et al. Exercise-based cardiac rehabilitation for coronary heart disease. Cochrane Database Syst Rev 2016;(1):CD001800.

16. Ben Gal T, Piepoli MF, Corrà U, et al. Exercise programs for LVAD supported patients: a snapshot from the ESC affiliated countries. Int J Cardiol 2015;201:215-9.

17. Racca V, Castiglioni P, Panzarino C, et al. End-stage heart failure: two surgical approaches with different rehabilitative outcomes. PLoS One 2017;12:e185717.

18. Ambrosetti M, Doherty P, Faggiano P, et al. Characteristics of structured physical training currently provided in cardiac patients: insights from the Exercise Training in Cardiac Rehabilitation (ETCR) Italian survey. Monaldi Arch Chest Dis 2017;87:778.

19. Jung MH, Houston B, Russell SD, Gustafsson F. Pump speed modulations and sub-maximal exercise tolerance in left ventricular assist device recipients: a double-blind, randomized trial. J Heart Lung Transplant 2017;36:36-41.

20. Mettauer B, Geny B, Lonsdorfer-Wolf E, et al. Exercise training with a heart device: a hemodynamic, metabolic, and hormonal study. Med Sci Sports Exerc 2001;33:2-8.
21. Balachandran S, Lee A, Royse A, et al. Upper limb exercise prescription following cardiac surgery via median sternotomy: a web survey. J Cardiopulm Rehabil Prev 2014;34:390-5.

22. Tuyl LJ, Mackney JH, Johnston CL. Management of sternal precautions following median sternotomy by physical therapists in Australia: a web-based survey. Phys Ther 2012;92:83-97.

23. Katijjahbe MA, Denehy L, Granger CL, et al. The sternal management accelerated recovery trial (S.M.A.R.T.) - standard restrictive versus an intervention of modified sternal precautions following cardiac surgery via median sternotomy: study protocol for a randomised controlled trial. Trials 2017;18:290.

24. Adams J, Cline MJ, Hubbard M, et al. A new paradigm for post-cardiac event resistance exercise guidelines. Am J Cardiol 2006;97:281-6.

25. Ueno A, Tomizawa Y. Cardiac rehabilitation and artificial heart devices. J Artif Organs 2009;12:90-7.

26. Compostella L, Russo N, Setzu T, et al. A practical review for cardiac rehabilitation professionals of continuous-flow left ventricular assist devices: historical and current perspectives. J Cardiopulm Rehabil Prev 2015;35:301-11.

27. Fattirolli F, Bonacchi M, Bugisser C, et al. Cardiac rehabilitation of patients with left ventricular assist device as destination therapy. Monaldi Arch Chest Dis 2009;72:190-9.

28. Jung MH, Gustafsson F. Exercise in heart failure patients supported with a left ventricular assist device. J Heart Lung Transplant 2015;34:489-496.

29. Compostella L, Polastri M, Lamotte M, Bellotto F, Antoine M. Physiotherapy and rehabilitation management in adult LVAD patients. In: Montalto A, Loforte A, Musumeci F, Krabatsch T, Slaughter MS, editors. Mechanical circulatory support in endstage heart failure. A practical guide. Cham; Springer: 2017. p. 412-414.

30. Bruce CR, Kostick KM, Delgado ED, et al. Reasons why eligible candidates decline left ventricular assist device placement. J Card Fail 2015;21:835-9.

31. Teochari AC, Michalopoulos G, Oikonomou EK, et al. Heart transplantation versus left ventricular assist devices as destination therapy or bridge to transplantation for 1-year mortality: a systematic review and meta-analysis. Ann Cardiothorac Surg 2018;7:3-11.

32. Reineke DC, Mohacsi PJ. New role of ventricular assist devices as bridge to transplantation: European perspective. Curr Opin Organ Transplant 2017;22:225-30. 